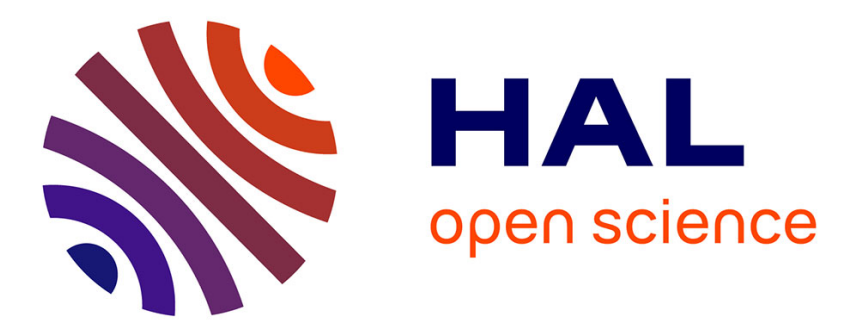

\title{
Design and implementation of a page-oriented "holographic" memory based on a Lippmann architecture
}

Kevin Contreras, Gilles Pauliat

\section{- To cite this version:}

Kevin Contreras, Gilles Pauliat. Design and implementation of a page-oriented "holographic" memory based on a Lippmann architecture. Applied optics, 2011, 50 (30), pp.5816-5823. hal-00632339

\section{HAL Id: hal-00632339 \\ https://hal-iogs.archives-ouvertes.fr/hal-00632339}

Submitted on 14 Oct 2011

HAL is a multi-disciplinary open access archive for the deposit and dissemination of scientific research documents, whether they are published or not. The documents may come from teaching and research institutions in France or abroad, or from public or private research centers.
L'archive ouverte pluridisciplinaire HAL, est destinée au dépôt et à la diffusion de documents scientifiques de niveau recherche, publiés ou non, émanant des établissements d'enseignement et de recherche français ou étrangers, des laboratoires publics ou privés. 


\title{
Design and implementation of a page-oriented
}

\section{«holographic» memory based on a Lippmann architecture}

\author{
Kevin Contreras, Gilles Pauliat* \\ Laboratoire Laboratoire Charles Fabry de l'Institut d'Optique, Univ Paris Sud, \\ Campus Polytechnique RD 128, 91127 Palaiseau cedex, France \\ *Corresponding author: gilles.pauliat@institutoptique.fr
}

Optical data storage inspired by Lippmann interference color photography was proposed a long time ago as an alternative to holographic memories. Very high capacities were predicted for a page-oriented approach with wavelength-multiplexing, but, up to now, such an architecture has never been implemented. Based on simple conception rules, we build such a page-oriented Lippmann data storage system. Sub-micrometer resolved data pages recorded in thick materials are evidenced by the experimental results.

OCIS codes: $090.4220,210.2860,090.7330$

\section{Introduction}

Holographic data storage is often thought as a possible replacement technology for the high capacity optical disks, CD, DVD and BR. Very impressive realizations are already close to a complete achievement [1]. Nevertheless various holographic arrangements exist and many are still on the race. Some of them are based on a bit oriented approach: bits of data are stored by recording micro-holograms in the focus of two counter-propagating beams [2-3]. Although large 
capacities are expected with these bit-oriented systems [4,5], we are interested in page-oriented approaches for which parallelism is thought to provide very high data rates [6]. In these architectures, data pages are imprinted on an image beam that is subsequently recorded by holography in the volume of the recording material by interfering it with a reference beam. Thanks to Bragg selectivity, several pages of data can be recorded in the same volume using a multiplexing procedure. The most commonly used is angular-multiplexing and its derivatives such as shift or phase-coded multiplexings [1,7-10]. Nevertheless, wavelength-multiplexing in which a new page is recorded at a different wavelength has also been investigated [11]. The most common configuration for holographic wavelength-multiplexing corresponds to a configuration in which the image beam and the reference beam enter the holographic recording medium by two opposite faces. This arrangement is known as Denisyuk holography, technique also often employed for color reproductions of artworks in museography [12].

Besides these holographic approaches, data storage architectures inspired from the principle of Lippmann interference color photography were proposed a long time ago. Incidentally Denisyuk holography was inspired by Lippmann photography [13]. In these Lippmann architectures, the incident image beam goes through the recording medium and is reflected back by a mirror set in contact with this recording medium, the image plane coincides with the mirror plane. The incident and reflected beams interfere and record a complex Bragg structure in the recording material. After this recording step, the mirror is removed and the material is processed. Illuminating the Bragg structure with white light diffracts the recorded image with its whole original wavelength spectrum. For color photographies, all spectral components are recorded at once, while for data storage, images at different wavelengths are usually recorded sequentially and correspond to different sets of data. 
Lippmann photography and Denisyuk holography share several common features. They both rely on the recording of interferences between counter-propagating waves and exploit the wavelength Bragg selectivity to record multiple data at the same location. The main difference originates from the reference beam. In Lippmann architectures, the image beam itself acts as the reference beam. There is thus no external phase reference and this is the reason why phase information cannot be recorded. Although recording phase information can slightly increase the capacity of holographic memories [14,15], most often this information is useless and the reconstructed pages are grabbed with an intensity detector. Therefore the intensity-coded data pages recorded in a Lippmann system should be adequate for data storage.

The first report on data storage inspired from Lippmann's ideas dates from 1965 [16]. From then, several variants have been investigated [17-20]. In all these demonstrations light corresponding to the adjacent data pixels does not interfere, either because these pixels are sequentially recorded or because the material thickness is smaller than the depth of field of the image. These architectures can thus be said as being bit-oriented. It has been shown that the capacity of these variants is relatively limited and does not benefit from the material thickness [21]. To get higher capacities, similar to those obtained with conventional holographic approaches, one should switch to a page-oriented approach as predicted in [21]. Besides these anticipated high capacities, the research on Lippmann data storage is further motivated by other expected advantages such as:

- A simple recording architecture;

- A large stability of this recording set-up;

- Required coherence lengths larger than twice the recording layer thickness only;

- An easy replication of the data from one master disk to a blank one; 
- A simple implementation of a homodyne detection for increasing the diffracted signals.

In this paper, we thus investigate such a page-oriented wavelength-multiplexed architecture. First, from simple considerations of grating recordings, we define two simple rules

for the conception of a data storage system based on the Lippmann arrangement. Second, using these rules as a guide, we design a recording/readout optical set-up. Experimental results of highresolution data pages recorded in a thick material are then reported.

\section{Rules of conception}

\section{A. Fidelity problem}

In case the depth of field of the image is smaller than the recording material thickness, Lippmann photography may suffer from a relatively low fidelity of the reconstructed images. This problem can be evidenced by comparing Lippmann photography to Denisyuk holography. The recording and readout steps for these both techniques are illustrated in Fig. 1. Beside the image beam (whose electric field complex amplitude is noted $A$ ), Denisyuk holography makes use of a (usually) plane wave reference beam, noted here $O$, to record an interference pattern proportional to $O^{*} A$, see Fig. 1.a. It is well known that reading out this Denisyuk hologram with the reference beam, Fig. 1.b, reproduces the image beam with a high fidelity: $O O^{*} A \propto A$. This situation differs from Lippmann storage in which an incident beam, whose complex amplitude is noted $B$, interferes with its reflection $A$ onto a mirror, Fig. 1.c. This complex amplitude $B$ considerably differs from the plane wave reference beam used for reading out, once again noted 
$O$ as for Denisyuk holography, see Fig. 1.d. We decompose $B$ in a Fourier series as $B=O+\sum b_{p}$ where $O$ is the zero spatial order and $\sum b_{p}$ the sum over all other spatial orders; the zero spatial order is indeed identical to the reference beam used for reading out the data. The information is thus reconstructed with a plane wave proportional to $O$, so that the reconstructed amplitude is $O B^{*} A=O\left(O^{*}+\sum b_{p}^{*}\right) A$. The first term of this sum corresponds to the desired reconstructed data corresponding to the reflected image beam used for recording, $O O^{*} A \propto A$. The reconstructed wave also contains a second term corresponding to a large amount of spurious waves proportional to $O \sum b_{p}^{*} A$. These waves may correspond to noise and are absent from Denisyuk holography. Consequently, in general, we expect the fidelity of the retrieved image to be very low. This happens if no special care is taken for conceiving the optical set-up.

Using this plane wave decomposition, in the following we derive two simple rules for designing a page-oriented Lippmann system for minimizing this noise. These simple rules will also allow us to estimate the tolerances of the optical set-up.

\section{B. Analysis of Lippmann architectures for defining construction rules}

For a thin recording material, i.e. when the material thickness is smaller than the image depth of field, there is no fidelity problem for recording binary intensity images. Indeed for a dark pixel, there is no light at the corresponding location during recording. There is thus no grating and therefore the diffracted intensity vanishes for this location. For a bright pixel a grating is recorded, but it does not contain any information about the phase of the recording beam. Indeed this grating is recorded by a stationary fringe pattern resulting from the interference between the recording beam and its reflection onto a mirror. The position of the zero order fringe is fixed relatively to the mirror (typically at a distance of one quarter of a wavelength for a metallic 
mirror): the mirror acts as a phase locker. The phase of the reconstructed beam thus only depends on the phase of the readout beam and on the mirror position but not on the recording beam phase. Consequently, for a thin recording material, we conclude that the intensity of binary images is correctly reproduced although the phase is not.

Conversely, for a thick material, even if a pixel is dark in the Lippmann mirror plane, contributions of the diffracted light for this pixel may originate from parts of the recorded grating that are distant from the mirror by more than the depth of field. For simplicity, in analyzing the contribution of noise due to the material thickness, we are going to separate the Bragg matched diffraction processes from the unmatched processes and show that a Lippmann interference pattern can be described as the superimposition of two Denisyuk holograms and a set of parasitic gratings.

Let us once again break the complex amplitudes of the incident and reflected beams under the form:

$$
B=O+\sum b_{p} \text { and } \quad A=O^{\prime}+\sum a_{p}
$$

Amplitudes $O$ and $O^{\prime}$ represent the zero spatial orders of the incident beam $B$ and of its reflection $A . A$ is the amplitude of the image beam we would like to reconstruct. The interference pattern between these two beams can be decomposed in three sets of reflection gratings:

$$
B^{*} A=O^{*}\left(0.5 O^{\prime}+\sum a_{p}\right)+\left(0.5 O^{*}+\sum b_{q}^{*}\right) O^{\prime}+\sum a_{p} \sum b_{q}^{*} .
$$

This decomposition in Fourier series allows us to identify three different sets of recorded gratings produced by the interfering pattern $B^{*} A$ : 
1. Set \#1, $O^{*}\left(0.5 O^{\prime}+\sum a_{p}\right)$, corresponds to the recording of a first Denisyuk hologram between the zero order of the incident beam and a beam carrying the information of the original image beam $A$ but whose zero order is half the one of the original image beam A.

2. Similarly set \#2, $\left(0.5 O^{*}+\sum b_{q}^{*}\right) O^{\prime}$, corresponds to the recording of a second Denisyuk hologram between the zero order of the reflected image beam and a beam carrying the information of the beam $B$ but whose zero order is half the one of $B$.

3. Set \#3, $\sum a_{p} \sum b_{q}^{*}$, recorded by interfering all orders different from zero is a set of gratings that does not exist in conventional Denisyuk holography. These gratings can thus be considered as parasitic gratings.

All these gratings diffract light during the readout process when the material is illuminated by reference beam $O$. Once again we can identify three sets of diffracted beams:

1. Wave diffracted by set \#1 that is the first Denisyuk hologram. This wave corresponds to the original image beam $A$ except that its intensity levels are slightly modified because its zero order is half the one of the original image. We thus anticipate grey level distortions in the retrieved data. This diffracted image is at focus in the plane corresponding to the image plane of $A$ (referred to as "Image" in Fig. 1) .

2. Wave diffracted by set $\# 2$, that is the second Denisyuk hologram. This wave corresponds to the phase conjugated beam of the original beam $B$ with, once again, the intensity levels slightly modified because its zero order is half the one of the original image. This diffracted image is at focus in the plane corresponding to the image plane of $B$ (referred to as " $2^{\text {nd }}$ image" in Fig. 1). 
3. All waves diffracted by parasitic set \#3. These waves are not Bragg matched during the reading out by $O$. They are responsible for noise and speckle in the retrieved images.

From this simple analysis we can draw a first important conclusion. In order to retrieve the data from the diffracted light, the first Denisyuk hologram must exist, that is the original image should have a non-vanishing zero order.

In order to get rid of the noise originating form the parasitic gratings, one should select images, i.e. data sets, for which the strength of these parasitic gratings (set \#3) is much lower than the set of the Denisyuk holograms, that is images for which the zero order is much stronger than any other diffracted order. This can be achieved quite simply in a data storage system. Typically for binary images whose pixels are selected randomly between two equiprobable intensity values " 0 " and " 1 ", then half of the energy is contained in the zero order. The parasitic gratings become negligible compared to the Denisyuk component of the Lippmann gratings.

In such a case, the main contribution to the diffracted light originates from the two Denisyuk holograms. As detailed above, diffraction on these two holograms replays the two waves $A$ and $B^{*}$, with slight grey level distortions. These two waves propagate in the same direction and carry the same set of data. To do so that they are focused in the same plane, one should do so that, during recording, the image plane exactly coincides with the Lippmann mirror.

In addition, if the wave fronts of these images are flat in this mirror plane (no phase information), then $A$ and $B$ are phase conjugated, i.e. $A \propto B^{*}$. The mirror acts as a phase locker and the two diffracted images interfere constructively.

All these conclusions are supported by the computer simulations we have conducted with a computer software described previously [21-22]. 
These simple considerations for designing a Lippmann storage system are summarized by these two following construction rules:

1. Rule \#1: Record pure amplitude images with a uniform phase.

2. Rule \#2: Place the Lippmann mirror in the image plane with an accuracy equal or better than the depth of field of the image.

It is important to note that the Rule \#1 not only imposes to use pure amplitude images, but also necessitates conceiving an optical system whose exit pupil is set ad infinitum relatively to the Lippmann mirror.

\section{Experimental realization}

\section{A. Optical set-up}

The scheme of our optical set-up is depicted in Fig. 2. Its photograph is shown in Fig. 3. It is fed by a single mode polarization preserving fiber. The diverging beam issued from this fiber freely propagates and goes through the image mask, mask on which is displayed the data page. It is then collected by a first video lens, turned by a polarizing beam splitter cube and then passes through a microscope objective. This microscope objective images the mask onto the Lippmann mirror. The video lens images the fiber output in the entrance pupil of the microscope objective. This ensures that the wave front of the beam in the mirror plane is totally flat in spite of the curved wavefront of the beam on the image mask. If, in addition, we use pure amplitude masks to input the data pages, then the construction Rule \#1 is fulfilled.

Typically, when the amplitude mask is removed during the readout procedure, the reading beam is a plane wave at normal incidence. The diffracted beam passes through the 
microscope objective and, thanks to the combination of a quarter-wave plate (not shown in Fig.2) with the polarizing beam splitter cube, recorded data are imaged onto the CCD camera by use of a second video lens.

The two identical video lenses are from Pentax (Model C7528-M, focal length $75 \mathrm{~mm}$ ). We selected a microscope objective to minimize the field curvature and thus to remain within the specifications given by our Rule \#2. It is a Plan Fluor ELWD 40xC from Nikon whose focal length is $5 \mathrm{~mm}$. Its correction ring allows correcting for the spherical aberrations introduced by the presence of the thick recording layer on its glass substrate. We designed the system so as the numerical aperture of the whole imaging system corresponds to the one of the microscope objective that is equal to 0.6 . Typically, with this numerical aperture at the wavelength of $532 \mathrm{~nm}$, the diameter of the Airy pattern at first nulls is $1.08 \mu \mathrm{m}$.

The CCD camera is an AVT Marlin F-201B with a 1/1.8" CCD sensor from Sony. It is made of $1628 \times 1236$ square pixels of $4.4 \times 4.4 \mu^{2}$. This sensor surface corresponds to an observed image area in the Lippmann mirror plane of $478 \times 363 \mu \mathrm{m}^{2}$.

The focus of the microscope objective is adjusted by a piezoelectric translation stage. The Lippmann recording substrate is mounted on a 2 -axis tilt platform. With this three-axis adjustment we can make the image plane coincide with the Lippmann mirror with an accuracy better than $2 \mu \mathrm{m}$ over the whole image field.

Ideally, a tunable laser source should be used to record the gratings. Because we do not possess such a source, instead, we used three lasers at three fixed wavelengths: 1) a single longitudinal mode, frequency doubled $\mathrm{Nd}: \mathrm{YVO}_{4}$ laser at $532 \mathrm{~nm} ; 2$ ) a multimode diode laser at $475 \mathrm{~nm} ; 3)$ a multimode diode laser at $650 \mathrm{~nm}$. The two first ones are used for wavelengthmultiplexing, the last one in the red is used for the alignment procedure, the recording material 
being inactinic in red light. All three wavelengths are injected in the single mode fiber and are selected by a set of shutters. The coherence lengths of the two first lasers, green and blue, are much larger than twice the recording layer thickness, which is sufficient for Lippmann recording.

\section{B. Recording substrate}

We selected a recording material considering two antagonist requirements for the Bragg wavelength selectivity:

- it should be strong enough to readout two superimposed gratings recorded at the two wavelengths of $475 \mathrm{~nm}$ and $532 \mathrm{~nm}$ without crosstalk;

- it should nevertheless be loose enough to allow the readout at the recording wavelength in spite of the unavoidable moderate shrinkage of the recording material.

We have selected a silver halide holographic gelatin whose thickness is about $9 \pm 3 \mu \mathrm{m}$ [23] Taking into account the gelatin refractive index, this thickness corresponds to a Bragg selectivity (full-width of half maximum for the amplitude) of about $\Delta \lambda_{B} \approx 10 \mathrm{~nm}$. As required, this value is smaller than the recording wavelength difference, $57 \mathrm{~nm}$, but large enough to accommodate for the shrinkage of about $1 \%$ we have with these silver halide plates.

The structure of the recording substrate is depicted in Fig. 4. The sensitive gelatin layer is laid on a $1.6 \mathrm{~mm}$ thick BK7 glass substrate. On top of this structure is glued a $100 \mu \mathrm{m}$ thick, polycarbonate, achromatic quarter-wave plate. Although in our first recording tests we used an aluminum mirror set in contact with the gelatin with an index matching liquid, all results reported below were obtained by using the Fresnel reflection on the gelatin-air interface as the Lippmann mirror. In spite of lower diffraction efficiency, the use of this Fresnel reflection inherently ensures that the mirror is in contact with the gelatin. Due to the bad flatness of our 
glass substrate and gelatin layer we could not guarantee this contact with the aluminum mirror. About $2 \mathrm{~cm}$ below the gelatin-air interface, a light trapping velvet absorbs the transmitted light.

Material shrinkage occurs during the wet processing. We measured it by recording reflection gratings with planes waves. By monitoring the diffraction efficiency versus the wavelength of the chemically processed plates with a Cary 14 spectrophotometer, we measured a shrinkage of about $1 \%$. These gratings can thus be readout at the recording wavelength, as desired for our demonstration.

Before reading out the recorded structure, the gelatin surface is coated by a black absorbing paint in order to remove the Fresnel reflection.

\section{Data pages and masks}

Ideally, the data pages should be imprinted on the optical beam by use of a spatial light modulator. For testing our set-up, we prefer to use chromium masks on a glass substrate. Due to their relatively low cost, chromium masks are indeed totally versatile; the pixel pitch can be changed arbitrarily by changing the mask. Furthermore, their ON/OFF ratio of intensity is much larger than the dynamic range of the CCD camera. With these chromium masks we insure that the measured signal to noise ratio of the retrieved image is neither limited by the contrast ratio of the spatial light modulator, nor by its flicker.

As described in the previous paragraph "Analysis of Lippmann architectures" we anticipate moderate contrasts in the retrieved images and grey level distortions as well. Therefore, our data pages are encoded with binary pixels $(\mathrm{ON}=$ transparent and $\mathrm{OFF}=$ opaque $)$ using bloc coding $[24,25]$. We use a $(3,16)$ coding, that is each code (from 0 to 255 ) of each ASCII character (Byte) of the file to be encoded on the data page is represented by a bloc of $4 \times 4$ pixels among which 3 are on the ON state. A typical data page is shown in Fig. 5. It is 
divided into sub-pages in the center of which the square feature is a fiducial pattern used to estimate, during the decoding of the diffracted image, the image spatial distortions (magnification, rotation and shifts). These patterns thus allow compensating for these distortions. Once these distortions are compensated, for each bloc, we determine the 3 pixels bearing the highest signals and thus attribute an ASCII value for this bloc. Comparing the decoded ASCII file with the known original ASCII file, we calculate the bloc error rate, BLER, defined as the ratio of the number of erroneous blocs to the total number of blocs.

In order to make its features visible for the reader, the resolution of the page shown in Fig. 5 is low. It contains $40 \times 32$ blocs only. However we tested our set-up with data pages of higher resolutions, up to $128 \times 96$ blocs, whose information capacity is $11.5 \mathrm{kByte}$, discarding the fiducial patterns. For these high-resolution masks, the pixel pitch is $0.88 \mu \mathrm{m}$ in the Lippmann mirror plane.

Replacing the recording substrate by an aluminum mirror, we checked the quality of our set-up with the high-resolution image detected with the CCD camera after being reflected on the mirror, but without any recording. We find a bloc error rate of $B L E R \approx 0.01$ corresponding only to errors located on the edges of the $11.5 \mathrm{kByte}$ page, thus demonstrating the optical quality of our set-up.

\section{Experimental results and analyses}

In Fig. 6, we show a data page recorded and detected at $475 \mathrm{~nm}$. The pixel pitch of the data mask is $2.64 \mu \mathrm{m}$. This corresponds to an image depth of field of $20 \mu \mathrm{m}$ that is larger than the recording material thickness. The retrieved image quality is high. For sake of comparison, the original data mask is the one previously shown in Fig. 5. We got a bloc error rate of 
$B L E R \approx 810^{-4}$ corresponding to a single error due to a single speck of dust. We checked that for this resolution absolutely no cross-talk is visible between two images superimposed at the same location and multiplexed at the two wavelengths $475 \mathrm{~nm}$ and $532 \mathrm{~nm}$. Thanks to the wavelength Bragg selectivity, they can be retrieved without ambiguity at their recording wavelength.

We also tested our set-up with the highest available resolution that is with a data mask whose pixel pitch is $0.88 \mu \mathrm{m}$. This pixel pitch is smaller than the diameter of the Airy pattern whose diameter is $1.08 \mu \mathrm{m}$ at the wavelength of $532 \mathrm{~nm}$. The depth of field of the image is now about $2 \mu \mathrm{m}$. This value is now much smaller than the holographic layer thickness. After recording such a data page at $532 \mathrm{~nm}$ and processing the plate, we obtained during the readout process, the raw image shown in Fig. 7. After decoding, we found that the error rates are quite non-uniform among this page. Some sub-pages are relatively well decoded while for other ones the error rate remains high. We attribute these inconsistent results to variations in the thicknesses of the plastic quarter-wave plate and of the holographic emulsion. Indeed because of these variations in thickness, it is not possible to exactly make the image plane coincide with the mirror plane (in practice, the interface "gelatin layer - air") over the whole image field. Our conception Rule \#2 cannot thus be fulfilled all over this field. This explanation for our relatively inconsistent results is supported by our computer simulations: as soon as the image plane moves away from the mirror plane by more than or about $2 \mu \mathrm{m}$, the detected images are blurred and cannot be decoded.

In spite of this problem whose origin is now identified, some of the sub-pages are correctly decoded. They correspond to locations where the focus is correct. For instance in Fig. 8.a, we show one of the sub-pages of the image detected by the CCD camera. In this image, the image pixel pitch is about $0.88 \mu \mathrm{m}$ in the mirror plane. Taking into account the $\mathrm{x} 15$ 
magnification of the optical system, this value corresponds to $13.3 \mu \mathrm{m}$ in the CCD plane, that is 3 times the size of a CCD pixel. After compensation of the spatial distortions (rotation, magnification and shift) determined from the positions of the fiducial patterns in all sub-pages, the average value of an ensemble of $3 \times 3$ pixels of the camera is attributed to the value of one pixel of the data image. The shifts are compensated within one CCD pixel; we did not employ any optimized sophisticated resampling technique that could further enhance the performances of our system [26]. We thus obtain the grey level image shown in Fig. 8.b. In each bloc made of $4 \times 4$ pixels we then seek for the 3 pixels bearing the highest values. These 3 pixels are then set to "1" and all others to " 0 ". This binarized image is shown in Fig.8.c. It has to be compared to the original sub-page shown in Fig. 8.d.

The decoded sub-page, Fig. 8.c, is thus very similar to the original one, Fig. 8.d. A detailed comparison demonstrates that they differ by only $3 \%$ of the bits of the image data, corresponding to a bloc error rate of $B L E R \approx 0.15$. This value for a bloc error rate is relatively large and should be compensated by the use of efficient error correction codes. Typically, the use of two successive error correction codes, the first on the initial data stream to be distributed between several pages and the second at the page level, should be used to reduce the bit error rate on the user file much below $10^{-12}$, value compatible with a memory capacity of a few TeraBytes [1].

Some of these remaining errors originate from small specks of dust and are thus not inherent from the Lippmann technique. This non-perfect bloc error rate should thus be improved with a cleaner material and/or by using a material with higher diffraction efficiency. Under our experimental conditions, the diffraction efficiency is indeed about $1 \%$ : scattered light thus greatly contributes to the relatively low quality of the images. 
Nevertheless these results clearly demonstrate that high-resolution data pages can be recorded in thick holographic media using the Lippmann principle. We are indeed very close to the theoretical resolution of the imaging system: the selected pixel pitch is smaller than the Airy pattern diameter. Furthermore, the error rate is mainly limited by imperfections and should be easily improved with a better recording substrate.

We checked that, together with this high resolution, the Bragg selectivity of the recorded grating is high enough for wavelength multiplexed images. Typically, reading out at $475 \mathrm{~nm}$ the grating corresponding to the image shown in Fig. 7 does not give any observable signal (i.e. signal above the noise level).

\section{Conclusion}

We have defined two simple construction rules for designing a page-oriented Lippmann data storage system. From these rules we anticipated the correct operation of such a storage system although distortions of the grey levels of the retrieved images are expected. Using these rules we have built an optimized recording set-up. With this system we have indeed demonstrated that the resolution of the data pages is just limited by the numerical aperture of the optical system: data pages with a pixel pitch of $0.88 \mu \mathrm{m}$ were retrieved in an optical system whose Airy disk diameter is $1.08 \mu \mathrm{m}$ and for which the image depth of field is smaller than the recording material thickness. At these high resolutions, the Bragg wavelength selectivity is preserved.

The price to pay for the apparent simplicity of the optical set-up (absence of any external reference beam) is a very severe requirement on its design and adjustment: the image plane should coincide with the Lippmann mirror over the whole image field with an accuracy better than the image depth of field. 
These analyses and experimental results corroborate the previous conclusions of computer simulations that predicted that the data capacity of Lippmann storage is comparable with the capacity of more conventional holographic approaches provided that special care is taken during the conception and adjustment of the experimental set-up [21]. These new results should thus contribute to the renewal of interest for this elegant technique which presents an enormous potential for high capacity storage.

\section{Acknowledgments:}

K. Contreras gratefully acknowledges the support of the Programme Alban, the European Union Programme of High Level Scholarship for Latin America (E07D401978PE). The research described here has been supported by Triangle de la physique contract MIMEA.

\section{References}

1. K. Curtis, L. Dhar, A. Hill, W. Wilson, and M. Ayres, Holographic Data Storage: From Theory to Practical Systems (John Wiley \& Sons, 2010).

2. H.J. Eichler, P. Kuemmel, S. Orlic, and A. Wappelt, "High-density disk storage by multiplexed microholograms," in IEEE J. Sel. Top. Quantum Electron. 4, 840-848 (1998).

3. R.R. McLeod, A.J. Daiber, M.E. McDonald, T.L. Robertson, T. Slagle, S.L. Sochava, and L. Hesselink, "Microholographic multilayer optical disk data storage," Appl. Opt. 44, 31973207 (2005). 
4. T. Tanaka, and S. Kawata, "Comparison of recording densities in three-dimensional optical storage systems: multilayered bit recording versus angularly multiplexed holographic recording," J. Opt. Soc. Am. A 13, 935-942 (1996).

5. R.R. McLeod, "Impact of phase aberrations caused by multilayer optical data storage in weakly inhomogeneous media," J. Opt. Soc. Am. B 26, 308-317 (2009).

6. S.S. Orlov, W. Phillips, E. Bjornson, Y. Takashima, P. Sundaram, L. Hesselink, R. Okas, D. Kwan, and R. Snyder, "High-Transfer-Rate High-Capacity Holographic Disk Data-Storage System," Appl. Opt. 43, 4902-4914 (2004).

7. K. Tanaka, M. Hara, K. Tokuyama, K. Hirooka, K. Ishioka, A. Fukumoto, and K. Watanabe, "Improved performance in coaxial holographic data recording", Opt. Express 15, 1619616209 (2007).

8. H. Horimai, X. Tan, and J Li, "Collinear holography," Appl. Opt. 44, 2575-2579 (2005).

9. Y. Taketomi, J. E. Ford, H. Sasaki, J. Ma, Y. Fainman, and S. H. Lee, "Incremental recording for photorefractive hologram multiplexing," Opt. Lett. 16, 1774-1776 (1991).

10. C. Denz, G. Pauliat, and G. Roosen, "Volume hologram multiplexing using a deterministic phase encoding method," Opt. Commun. 85, 171-176 (1991).

11. G.A. Rakuljic, V. Leyva, and A. Yariv, "Optical data storage using orthogonal wavelength multiplexed volume hologram," Opt. Lett. 17, 1471-1473 (1992).

12. Y. Denisyuk, "The imaging of the optical properties of an object in a wave field of radiation scattered by it," Opt. Spectrosc. 15, 279-284 (1963).

13. Y.N. Denisyuk, "Imaging properties of light intensity waves: the development of the initial Lippmann ideas," J. Opt. 22, 275-280 (1991). 
14. B. Das, J. Joseph, and K. Singh, "Phase modulated gray-scale data pages for digital holographic data storage," Opt. Commun. 282, 2147-2154 (2009).

15. Z. Göröcs, G. Erdei, T. Sarkadi, F. Ujhelyi, J. Reményi, P. Koppa, and E. Lorincz, "Hybrid multinary modulation using a phase modulating spatial light modulator and a low-pass spatial filter," Opt. Lett. 32, 2336-2338 (2007).

16. H. Fleisher, P. Pengelly, J. Reynolds, R. Schools, and G. Sincerbox, "An optically accessed memory using the Lippmann process for information storage" in Optical and Electro-Optical Information Processing (MIT Press, 1965).

17. A.S. Hoffman, "Optical information storage in three-dimensional media using the Lippmann Technique," Appl. Optics 7, 1949-1954 (1968).

18. G. Maire, G. Pauliat, and G. Roosen, "Homodyne detection readout for bit-oriented holographic memories" Opt. Lett. 31, 175-177 (2006).

19. J.-J. Yang, and M.-R. Wang, "White light micrograting multiplexing for high density data storage" Opt. Lett. 31, 1304-1306 (2006).

20. F. Guattari, G. Maire, K. Contreras, C. Arnaud, G. Pauliat, G. Roosen, S. Jradi, and C. Carré, "Balanced homodyne detection of Bragg microgratings in photopolymer for data storage" Opt. Express 5, 2234-2243 (2007).

21. K. Contreras, G. Pauliat, C. Arnaud, and G. Roosen, "Application of Lippmann interference photography to data storage", J. Europ. Opt. Soc. Rap. Public. 08020 Vol 3 (2008).

22. G. Pauliat, and K. Contreras, "Experimental investigation of a page-oriented Lippmann "holographic" data storage system," Proc. SPIE- 7730, 773004 (2010).

23. Silver halide plate, Ultimate 08: http://www.ultimate-holography.com. 
24. G.W. Burr, J. Ashley, H. Coufal, R.K. Grygier, J.A. Hoffnagle, C.M. Jefferson, and B. Marcus, "Modulation coding for pixel-matched holographic data storage," Opt. Lett. 22, 639641 (1997).

25. B. Marcus, "Modulation Codes for Holographic Recording", in "Holographic Data Storage", H.J. Coufal, D. Psaltis, and G.T. Sincerbox, eds. (Springer Series in Optical Sciences, Springer-Verlag, 2000), pp. 283-292.

26. M. Ayres, A. Hoskins, and K. Curtis, "Image oversampling for page-oriented optical data storage," Appl. Opt. 45, 2459-2464 (2006). 


\section{Figure captions}

Fig. 1: a) Recording scheme for Denisyuk holography with $A$ and $O$ the image and reference beams; b) readout scheme for Denisyuk holography; c) recording scheme for Lippmann storage with image beam $A$ resulting from the reflection of the incident beam $B$ onto the mirror; d) readout scheme for Lippmann storage, the mirror is replaced by an absorbing layer. $e$ is the recording medium thickness.

Fig. 2: Optical scheme of the recording/readout set-up.

Fig. 3: Photograph of the optical set-up.

Fig. 4: Recording substrate arrangement. Incoming light is partly reflected by the interface air/sensitive layer; transmitted light is blocked by the light trapping velvet.

Fig. 5: Typical data page with $(3,16)$ bloc coding. This page is made of $5 \times 4$ sub-pages made each of $8 \times 8$ blocs. Fiducial patterns are located in the center of each sub-page.

Fig. 6: Image grabbed by the CCD camera corresponding to a data page recorded and retrieved at $475 \mathrm{~nm}$. The pixel pitch is $2.64 \mu \mathrm{m}$.

Fig. 7: Image grabbed by the CCD camera corresponding to a data page recorded and retrieved at $532 \mathrm{~nm}$ with the smallest pixel pitch of $0.88 \mu \mathrm{m}$.

Fig. 8: Decoding procedure of a sub-page recorded at $532 \mathrm{~nm}$, retrieved at $532 \mathrm{~nm}$ and detected with the CCD camera. The pixel pitch is $0.88 \mu \mathrm{m}$. a) Sub-page grabbed with the CCD camera without any processing; b) after compensation of distortions, rotation, magnification and shifts (see text); c) after decoding; d) comparison with the original sub-page before recording. 
Fig. 1

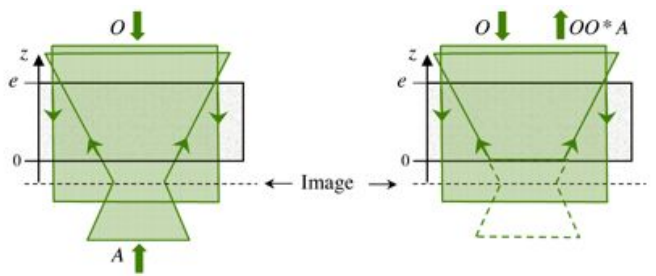

(a)

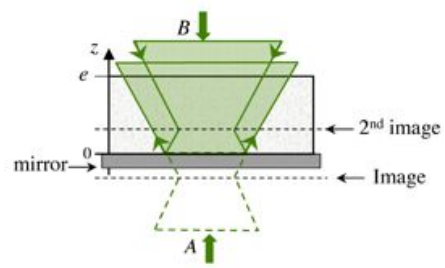

(c) (b)

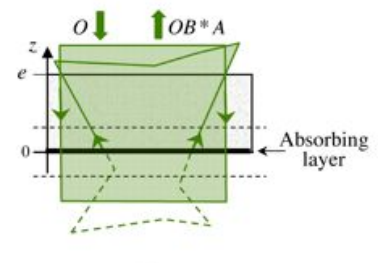

(d) 
Fig. 2

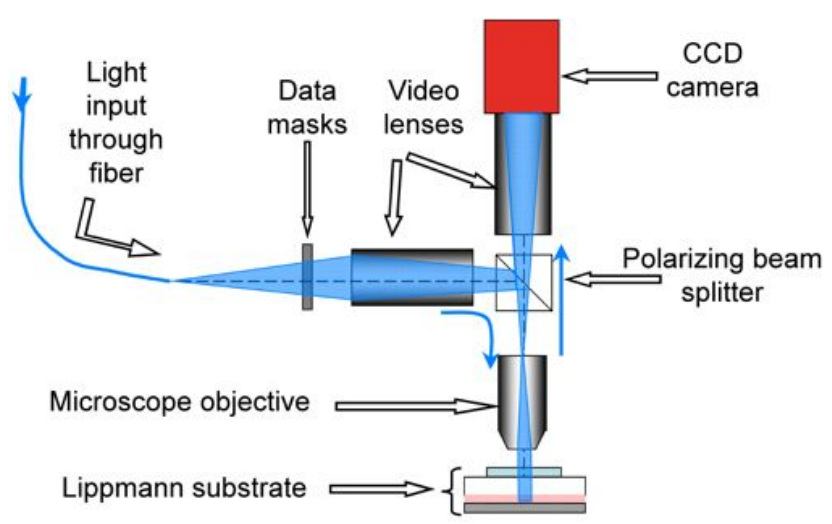


Fig. 3

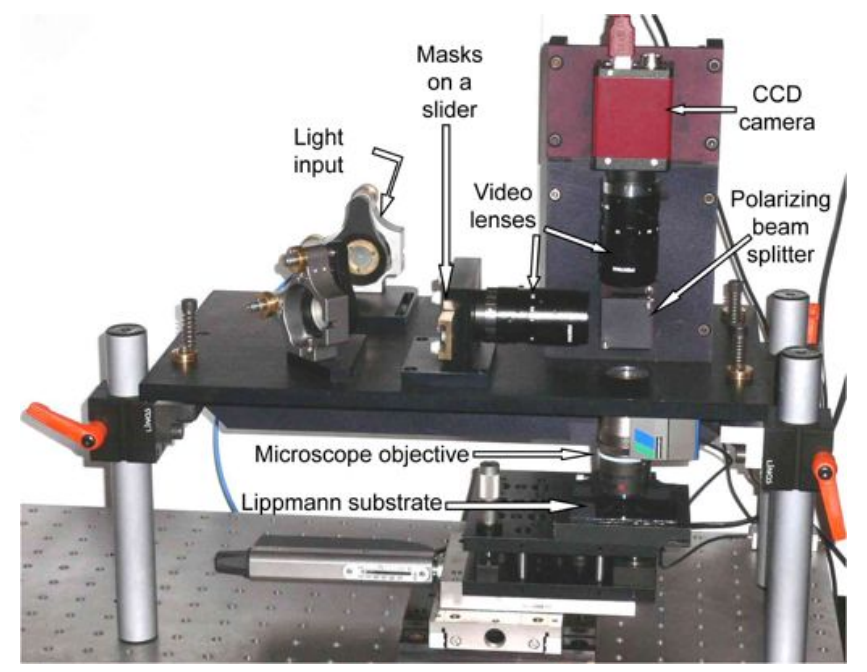


Fig. 4

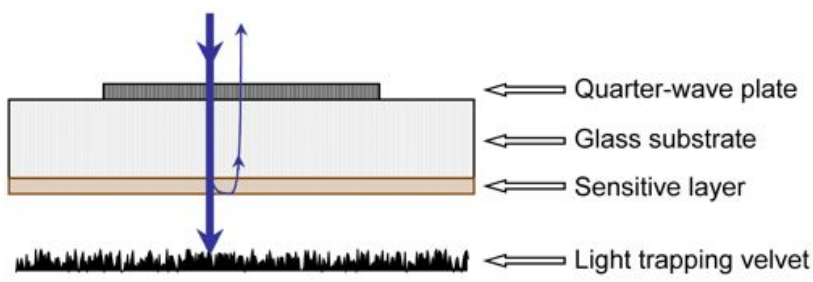


Fig. 5

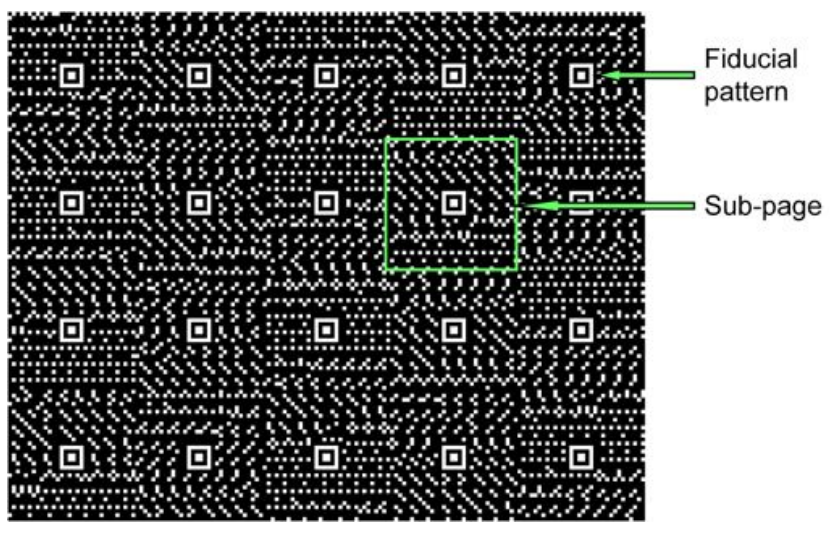


Fig. 6

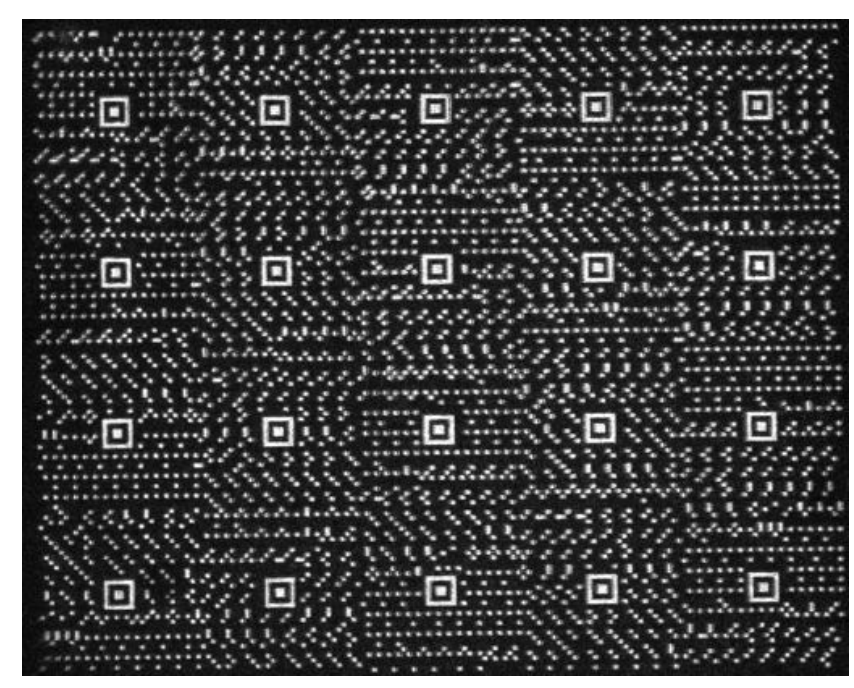


Fig. 7

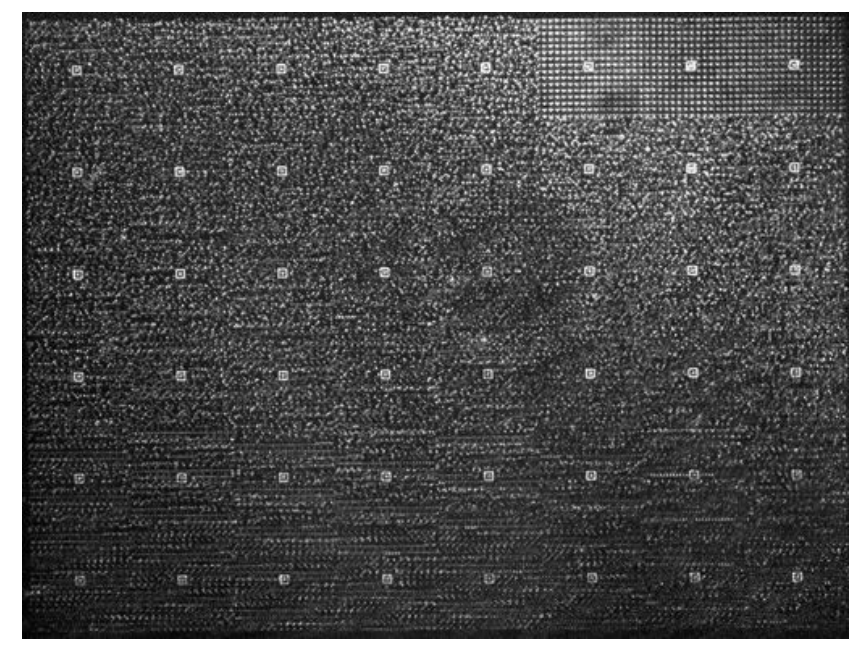


Fig. 8

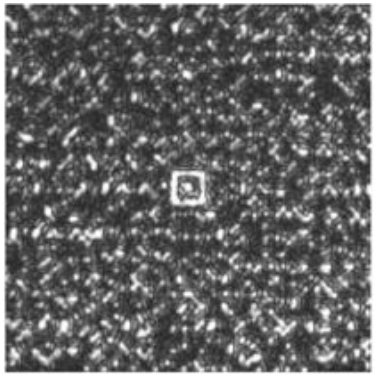

(a)

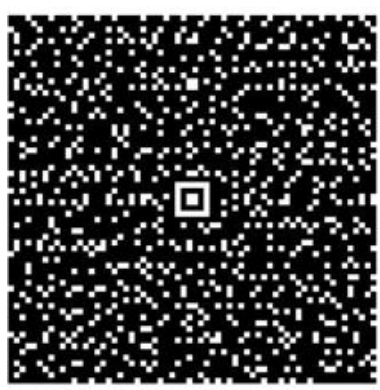

(c)

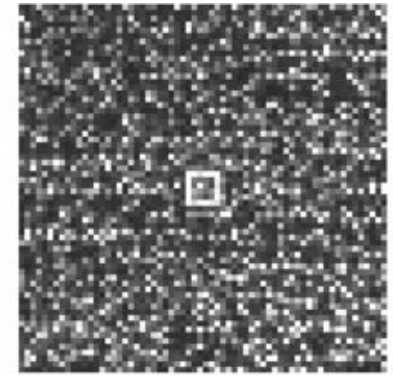

(b)

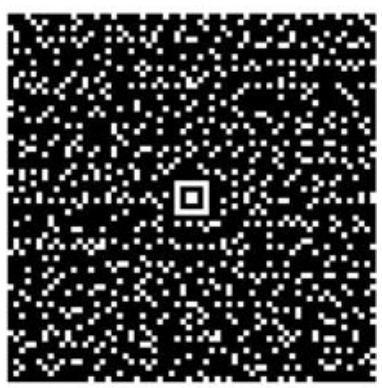

(d) 